\title{
Representação de ciclos harmônicos de séries temporais Modis para análise do cultivo da cana-de-açúcar
}

\author{
João Francisco Gonçalves Antunes(1), \\ Rubens Augusto de Camargo Lamparelli( ${ }^{(2)}$ e Luiz Henrique Antunes Rodrigues ${ }^{(3)}$
}

(1)Embrapa Informática Agropecuária, Avenida André Tosello, № 209, Campus da Unicamp, Caixa Postal 6041, CEP 13083-886 Campinas,
SP, Brasil. E-mail: joao.antunes@embrapa.br (2)Universidade de Campinas, Núcleo Interdisciplinar de Planejamento Energético, Cidade
Universitária Zeferino Vaz, CEP 13083-860 Campinas, SP, Brasil. E-mail: lamparel@unicamp.br (3)Universidade de Campinas, Faculdade de
Engenharia Agrícola, avenida Cândido Rondon, no 501, Barão Geraldo, CEP 13083-875 Campinas, SP, Brasil. E-mail: lique@feagri.unicamp.br

Resumo - O objetivo deste trabalho foi avaliar o cultivo da cana-de-açúcar, em análise harmônica aplicada a uma série temporal de índices de vegetação Modis, com a representação dos termos harmônicos. Os dados diários de precipitação pluvial para o Estado de São Paulo foram obtidos do Agritempo, acumulados para o período de 16 dias das composições Modis, ao longo das safras de 2004/2005 a 2011/2012. Utilizaram-se o índice de vegetação por diferença normalizada (NDVI) e o índice de realce da vegetação (EVI) em séries temporais decompostas em termos harmônicos pela análise harmônica. Para visualizar a condição de crescimento da vegetação de áreas agrícolas, em especial a informação de fase, aplicou-se a transformação HLS aos termos harmônicos obtidos com o algoritmo Hants, por meio do programa Envi. O cultivo da canade-açúcar no Estado de São Paulo apresenta padrões espaciais coerentes com o ciclo de desenvolvimento da cultura e consistentes com a variabilidade das precipitações sazonais que afetam diretamente o momento de máximo dos índices de vegetação. O pico vegetativo da cana-de-açúcar ocorre durante a fase de crescimento, em anos com chuvas normais; porém, em anos de pouca chuva, a fase de maturação da cultura é antecipada e, em anos de muita chuva, a fase de crescimento é antecipada, o que causa o atraso da maturação.

Termos para indexação: análise harmônica, índices de vegetação, processamento de imagens, sensoriamento remoto.

\section{Representation of harmonic cycles of Modis time series for the analysis of sugarcane cultivation}

\begin{abstract}
The objective of this work was to evaluate sugarcane cultivation, in a harmonic analysis applied to a time series of Modis vegetation indices, with the representation of harmonic terms. Daily rainfall data were obtained from Agritempo for the state of São Paulo, Brazil, and accumulated for a period of 16 days of Modis compositions, from the 2004/2005 to 2011/2012 crop seasons. The normalized difference vegetation index (NDVI) and the enhanced vegetation index (EVI) were used in time-series decomposed in harmonic terms by the harmonic analysis. In order to visualize the growing conditions of vegetation in agricultural areas, specially the phase information, the HLS transformation was applied to the harmonic terms obtained by the Hants algorithm, using Envi software. Sugarcane cultivation in the state of São Paulo shows spatial patterns that are coherent with the sugarcane development cycle and consistent with the variability of seasonal rainfall that directly affect the maximum period of vegetation indices. The peak growth stage of sugarcane occurs in years of normal rainfall; however, in years with below normal rainfall, sugarcane maturation phase is anticipated, and, in years with above normal rainfall, the growth phase is anticipated, which causes maturation delay.
\end{abstract}

Index terms: harmonic analysis, vegetation indices, image processing, remote sensing.

\section{Introdução}

O setor agrícola brasileiro está sendo marcado por um novo ciclo do plantio da cana-de-açúcar para obtenção do álcool combustível. Além de ser o maior produtor de cana-de-açúcar do mundo, o Brasil é também o maior exportador de açúcar e o segundo maior produtor de etanol. O Centro-Sul do país é responsável por $90 \%$ do total da produção nacional, e o Estado de São Paulo é o maior produtor, respondendo por mais de $60 \%$ de toda a produção de açúcar e etanol da região. Metade do açúcar produzido é exportada e

Pesq. agropec. bras., Brasília, v.51, n.11, p.1868-1879, nov. 2016

DOI: 10.1590/S0100-204X2016001100009 
tem uma participação aproximada de $35 \%$ na balança comercial. Além disso, a agroindústria sucroalcooleira representa 6\% dos empregos brasileiros (Unica, 2016).

A cana-de-açúcar é uma poácea e, em consequência de seu manejo, pode ser caracterizada como uma cultura semiperene, que permite de três a seis cortes para que posteriormente seja feita a reforma do canavial. Apresenta rápido crescimento, reprodução abundante e aproveitamento econômico de grande parte da planta. O ciclo fenológico da cultura é composto pelas fases de brotação, perfilhamento, crescimento e maturação (Scarpari \& Beauclair, 2010).

Do plantio até a primeira colheita, a cana-planta pode ter um ciclo de desenvolvimento com duração de 18 meses (cana de um ano e meio) ou 12 meses (cana de ano), a depender da época e da região de plantio. Após a primeira colheita, a cana-soca passa a ter um ciclo de 12 meses e, a cada corte, ocorre a rebrota da soqueira e o início de um novo ciclo. No Estado de São Paulo, predominam os ciclos de produção da cana de ano e meio, plantada de janeiro a maio, e da cana de ano, plantada de setembro a dezembro, e a colheita estende-se de abril a dezembro (Anjos \& Figueiredo, 2010).

Os satélites de monitoramento ambiental vêm operando de forma sistemática nos últimos anos, provendo uma visão sinóptica e frequente de grandes áreas, a custos baixos. As imagens geradas pelos sensores orbitais têm características que possibilitam o estudo e a análise do ciclo de desenvolvimento de culturas agrícolas (Vicente et al., 2012; Brown et al., 2013).

O sensor Modis - a bordo da plataforma orbital Terra do programa internacional EOS (Earth Observing System), liderado pela Nasa (National Aeronautics and Space Administration) - tem gerado dados processados para estudos globais da vegetação. O satélite Terra foi lançado em dezembro de 1999 e cruza com o Equador às 10:30 h (horário local), em órbita descendente da direção norte para sul (Soares et al., 2007).

Os dados Modis de moderada resolução espacial, elevada repetitividade temporal, boa qualidade radiométrica, alta precisão geométrica, com correção atmosférica e de distribuição gratuita têm características que representam um grande potencial de aplicação ao monitoramento da cultura da cana-deaçúcar (Freitas et al., 2011; Adami et al., 2012).
Os índices de vegetação obtidos dos dados Modis buscam realçar a resposta espectral das plantas e amenizar a influência do solo. Quando observados a partir de séries temporais, podem ser utilizados para distinguir a fenologia da vegetação e registrar as alterações do uso e da cobertura do solo (Wardlow et al., 2007; Verbesselt et al., 2010).

A análise harmônica vem sendo empregada no estudo de séries temporais de índices de vegetação, para examinar as mudanças que acontecem na superfície terrestre, a fim de encontrar evidências de alterações do desenvolvimento da vegetação e revelar padrões da dinâmica temporal (Brown et al., 2007; Jia et al., 2011). Pode ser utilizada para estudar o comportamento cíclico dos índices de vegetação, por meio da representação dos termos harmônicos, num espaço de cores que facilite a interpretação visual das imagens.

O objetivo deste trabalho foi avaliar o cultivo da cana-de-açúcar, por meio da análise harmônica aplicada à série temporal de índices de vegetação Modis, ao longo das safras 2004/2005 a 2011/2012, mediante a representação dos termos harmônicos.

\section{Material e Métodos}

O trabalho foi realizado no Estado de São Paulo, região Sudeste do Brasil, $19^{\circ} 40^{\prime} \mathrm{S}$ e $25^{\circ} 20^{\prime} \mathrm{S}$ e $44^{\circ} 10^{\prime} \mathrm{W}$ e $53^{\circ} 10^{\prime} \mathrm{W}$, que apresenta extensas áreas de cultivo da cana-de-açúcar.

As séries temporais de imagens do satélite Terra foram obtidas do banco de produtos Modis, na base estadual brasileira (Embrapa Informática Agropecuária, 2016), que armazena e disponibiliza os produtos na Internet, em recortes por estado, na projeção geográfica e no formato GeoTIFF (Esquerdo et al., 2010). As séries temporais completas do produto MOD13Q1 da coleção 5, de resolução espacial de 250 m estão disponíveis nesse banco de imagens, que consiste de composições dos pixels de alta qualidade radiométrica, melhor geometria de observação, menor presença de nuvens e aerossóis, selecionados das imagens diárias durante o período de 16 dias (Latorre et al., 2007), atualizadas conforme a distribuição do LP DAAC (Land Processes Distributed Active Archive Center) do EOS da Nasa, para todos os estados do Brasil.

Um dos índices de vegetação utilizado foi o índice de vegetação por diferença normalizada (NDVI) (Rouse

Pesq. agropec. bras., Brasília, v.51, n.11, p.1868-1879, nov. 2016 DOI: $10.1590 / \mathrm{S} 0100-204 X 2016001100009$ 
et al., 1974), para atenuar a interferência do solo na resposta espectral da vegetação e diminuir a influência da atmosfera e do ângulo de elevação solar em imagens temporais. O NDVI explora o contraste entre as bandas do vermelho e do infravermelho próximo, conforme equação a seguir, e varia de $-1,0 \mathrm{a}+1,0$ : NDVI $=\left(\rho_{\mathrm{IVP}}\right.$ $\left.\rho_{\mathrm{VER}}\right) /\left(\rho_{\mathrm{IVP}}+\rho_{\mathrm{VER}}\right)$, em que: $\rho_{\mathrm{IVP}}$ é o fator de refletância na banda do infravermelho próximo; e $\rho_{\mathrm{VER}}$ é o fator de refletância na banda do vermelho.

O outro índice de vegetação utilizado no trabalho foi o índice de realce da vegetação (EVI) (Huete et al., 1994), que otimiza a resposta espectral da vegetação e melhora a sensibilidade em regiões de maior biomassa, propiciando o monitoramento da vegetação por meio da redução dos efeitos de substrato do dossel vegetal e de influências atmosféricas. O EVI, que também varia de $-1,0$ a $+1,0$, representa melhor a dinâmica da vegetação, ao longo de uma série temporal de imagens, é calculado pela seguinte equação,

$$
\mathrm{EVI}=2,59 \times\left[\frac{\left(\rho_{\mathrm{IVP}}-\rho_{\mathrm{VER}}\right)}{\left(\rho_{\mathrm{IVP}}+6,0 \times \rho_{\mathrm{VER}}-7,5 \times \rho_{\mathrm{AZU}}+1\right)}\right],
$$

em que: $\rho_{\text {IVP }}$ é o fator de refletância na banda do infravermelho próximo; $\rho_{\mathrm{VER}}$ é o fator de refletância na banda do vermelho; $\rho_{\mathrm{AZU}}$ é o fator de refletância na banda do azul.

A análise harmônica foi utilizada com o propósito de decompor as séries temporais dos índices de vegetação EVI e NDVI em termos harmônicos, que são ondas cossenoidais ou senoidais, em que cada onda é definida por um valor único de amplitude e fase (Figura 1).
A amplitude corresponde à metade do valor em que a função é maximizada, e a fase é o deslocamento entre a origem e o pico da onda no intervalo de 0 até $2 \pi$. Cada termo harmônico representa o número de ciclos completados por uma onda, num determinado intervalo de tempo, e é responsável por um percentual da variância total da série temporal de dados original. Assim, o primeiro harmônico tem um período $\mathrm{T}$ igual ao período total, o segundo harmônico corresponde à metade do período do primeiro harmônico $\mathrm{T} / 2$, o terceiro harmônico a T/3 e assim sucessivamente (Jakubauskas et al., 2001).

Uma série temporal de dados de $\mathrm{n}$ amostras equidistantes pode ser representada pela transformada de Fourier, para a obtenção dos valores ao longo do tempo $t$, o que permite a reconstrução da curva, por meio da equação abaixo:

$$
\mathrm{y}_{\mathrm{t}}=\overline{\mathrm{c}}+\sum_{\mathrm{j}=1}^{\mathrm{n} / 2} \mathrm{c}_{\mathrm{j}} \cos \left(\frac{2 \pi \mathrm{t}}{\mathrm{n}}-\phi_{\mathrm{j}}\right)
$$

em que: c é a média dos valores da série temporal; $c_{\mathrm{j}}$ é a amplitude do j-ésimo harmônico; $\phi_{\mathrm{j}}$ é a fase do j-ésimo harmônico. A amplitude é calculada por:

$$
c_{j}=\sqrt{a_{j}^{2}+b_{j}^{2}},
$$

em que: $a_{j}$ e $b_{j}$ são as componentes de cosseno e seno do vetor amplitude $c_{j}$ e são obtidas pelas equações:

$$
\begin{aligned}
& a_{j}=\frac{2}{n}+\sum_{t=1}^{n} y_{t} \cos \left(\frac{2 \pi t}{n}\right), \text { para } t \geq 0 . \\
& b_{j}=\frac{2}{n}+\sum_{t=1}^{n} y_{t} \operatorname{sen}\left(\frac{2 \pi t}{n}\right), \text { para } t \geq 1 .
\end{aligned}
$$
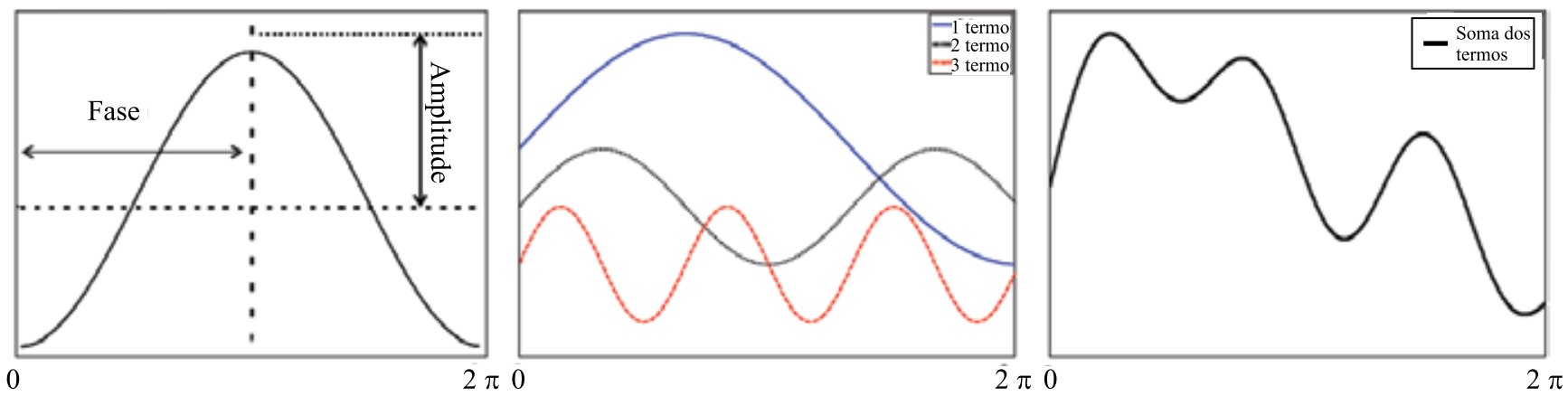

Figura 1. Representação dos termos harmônicos. Fonte: adaptado de Adami (2010). 
A fase é calculada pela seguinte equação:

$$
\phi_{j}= \begin{cases}\arctan \frac{b_{j}}{a_{j}}, & \text { se } a_{j}>0 \\ \arctan \frac{b_{j}}{a_{j}} \pm \pi, & \text { se } a_{j}<0 \\ \frac{\pi}{2}, & \text { se } a_{j}=0\end{cases}
$$

As imagens obtidas a partir da aplicação da transformada de Fourier foram geradas numa base por pixel para cada imagem da série temporal. Na prática, considerar um ciclo anual significa decompor a variação dos índices de vegetação em harmônicos que representem a média e as oscilações anuais, semestrais, quadrimestrais, etc., o que propicia a identificação de áreas agrícolas. A imagem harmônico zero (termo aditivo) corresponde à média dos índices de vegetação e representa o vigor total para cada tipo de cobertura vegetal, durante o período. As imagens amplitude indicam a variação máxima dos índices de vegetação para todo o período. A fase, com intervalo de valores de $0^{\circ}$ a $360^{\circ}$, indica o tempo ao longo da série temporal em que acontece o máximo valor de amplitude e pode ser associada aos meses do ano.

O comportamento desses parâmetros é indicativo do tipo de mudança que vem ocorrendo com o tempo. As mudanças sazonais somente na amplitude podem indicar variações do tipo de uso do solo ou das condições da vegetação. As mudanças apenas de fase podem indicar variações do tempo de máximo vigor vegetativo, que pode estar relacionado às variações da época de plantio ou de colheita. As mudanças da amplitude e da fase podem indicar transformações significativas na superfície terrestre, relacionadas à alteração do manejo do solo ou à substituição da vegetação.

Altos valores de amplitude indicam um alto grau de variação, e o harmônico onde essa variação acontece indica a periodicidade do evento. Elevados valores de amplitude do primeiro harmônico indicam uma cultura agrícola com padrão temporal unimodal e, no segundo harmônico, um padrão temporal bimodal, diferentemente de áreas de pastagens e de vegetação natural, cujos padrões temporais têm pequena variação (Jakubauskas et al., 2002).

O processamento das séries temporais de EVI e NDVI foi realizado por meio do programa
Hants (Harmonic analysis of NDVI time-series), desenvolvido originalmente por Roerink et al. (2000) e implementado em linguagem IDL (Interactive data language) por Wit \& Su (2005). O conceito básico do algoritmo é que o desenvolvimento da vegetação apresenta um forte efeito sazonal, que pode ser simulado por funções senoidais de baixa frequência, com diferentes fases e amplitudes. As nuvens provocam alterações nas séries temporais e podem ser consideradas ruídos de altas frequências. $\mathrm{O}$ algoritmo Hants analisa a série temporal original, identifica as áreas de baixas frequências referentes à vegetação e, a partir da transformada de Fourier, elimina os valores discrepantes da série temporal que representam altas frequências.

A representação visual eficaz das imagens amplitude e fase dos termos harmônicos pode auxiliar a interpretação das mudanças fenológicas da vegetação pela combinação de cores. Porém, a fase é quantificada numa escala circular, o que torna difícil a interpretação de imagens no espaço de cores RGB, em que ocorre uma mudança abrupta entre a cor escura e a cor brilhante. No entanto, no espaço de cores HLS (hue, lightness, saturation), a componente matiz é inerentemente circular e proporciona um efeito visual de transição suave do azul para o magenta e para o vermelho. Os outros parâmetros harmônicos são lineares e podem ser bem representados nas componentes luminosidade ou saturação (HallBeyer, 2007).

Com base nisso, para analisar a condição de crescimento da vegetação de áreas agrícolas, em especial a informação de fase, a transformação HLS foi aplicada aos termos harmônicos obtidos com o Hants, segundo o procedimento desenvolvido por Foley \& Van Dam (1984), que está disponível no programa Envi. Esse espaço de cores é formado por um duplo hexacone (Figura 2).

As cores primárias estão situadas no perímetro da base comum, e as cores branca e preta no vértice de cada um dos cones. Os tons de cinza localizam-se sobre o eixo comum dos dois cones. Matiz $(\mathrm{H})$ é o ângulo ao redor do eixo vertical do hexacone, percorrido no sentido anti-horário, com o vermelho em $0^{\circ}$, amarelo em $60^{\circ}$, verde em $120^{\circ}$, ciano em $180^{\circ}$, azul em $240^{\circ}$ e magenta em $300^{\circ}$. A saturação (S) é medida radialmente a partir do eixo vertical, em que zero são as cores brancas com os tons de cinza que variam até 
um referente às cores puras. A luminosidade (L) pode variar de zero para preto até um para branco, em que as cores puras apresentam luminosidade de 0,5.

A distribuição das chuvas durante a safra pode afetar os padrões fenológicos da cana-de-açúcar, ao longo
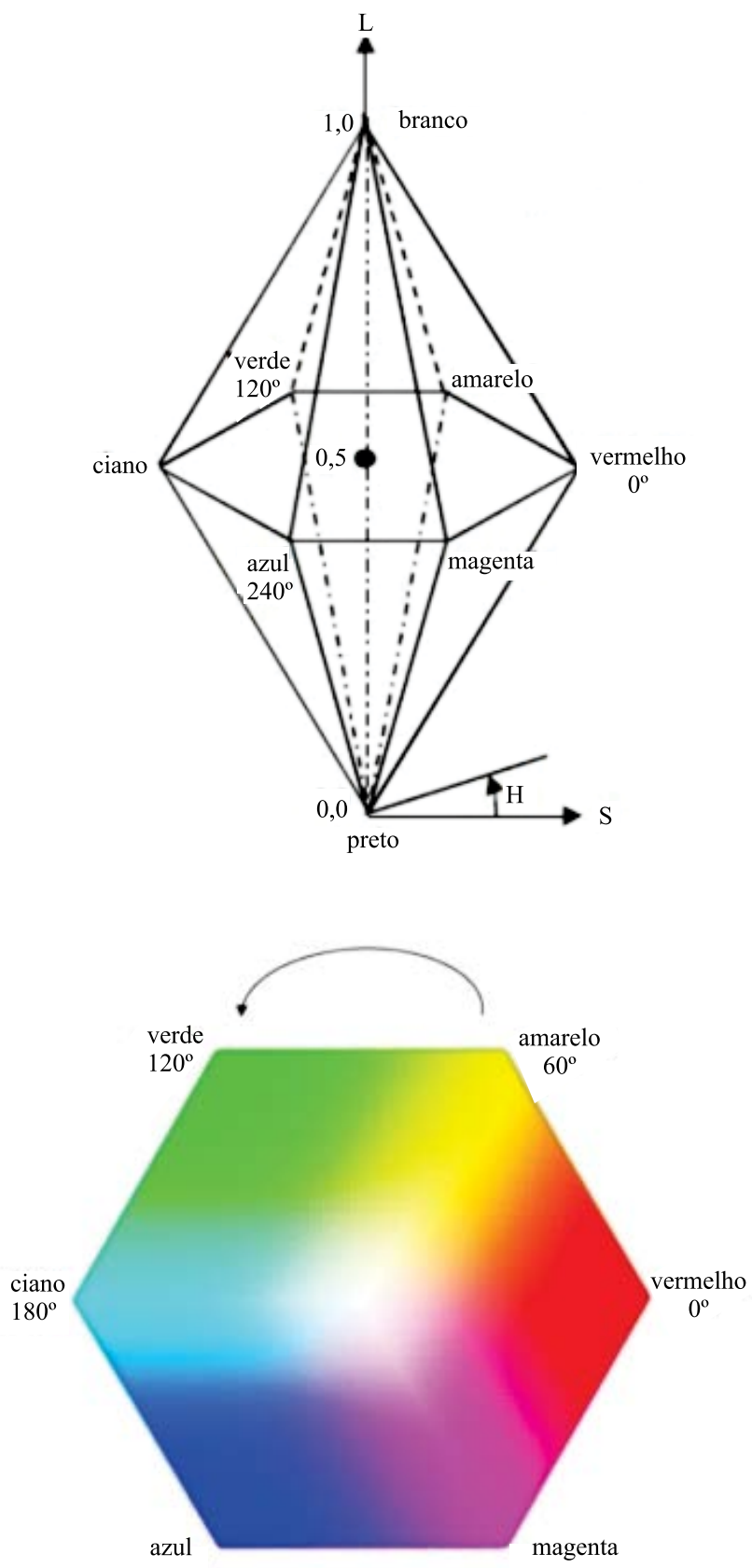

Figura 2. Espaço de cores HLS. Fonte: adaptado de Antunes et al. (2013). do seu ciclo de desenvolvimento e alterar o momento de maior vigor vegetativo. Para auxiliar a análise da dinâmica temporal da cana-de-açúcar, utilizaram-se os dados diários de precipitação pluvial do Estado de São Paulo, obtidos por meio do Agritempo (Agritempo, 2016), acumulados para o período de 16 dias das composições Modis, ao longo das safras de 2004/2005 a $2011 / 2012$.

\section{Resultados e Discussão}

Para a análise do cultivo da cana-de-açúcar, considerou-se o período iniciado em outubro do ano anterior ao plantio e terminado em setembro do ano da colheita, englobando, de forma geral, o ciclo fenológico característico da cultura, com duração de 12 meses, que predomina no Estado de São Paulo.

As séries temporais de EVI e NDVI do Modis foram decompostas pelo Hants, separadamente, com ênfase para o período de seis meses, de meados de setembro a meados de abril de cada safra, com 14 imagens de cada índice de vegetação que cobrem metade do ciclo fenológico da cana-de-açúcar, da fase de perfilhamento até o pico vegetativo na fase de crescimento, em que ocorre o alongamento dos colmos, com grande acúmulo de biomassa, e produção foliar abundante que proporcionam cobertura total do solo, indo para a fase de maturação, quando é iniciada a colheita, no total de 224 imagens processadas.

Os termos harmônicos gerados foram a amplitude e a fase para as frequências $0,1,2$ e 3, que correspondem à média do EVI e NDVI de cada série temporal e aos ciclos de seis, três e dois meses. Esses três harmônicos são responsáveis por explicar a maioria das variâncias das séries temporais dos índices de vegetação (Antunes et al., 2013). Os dados estão no fator original de escala, no intervalo de -2.000 a 10.000. A tolerância do erro para ajuste da curva - entre os valores originais e os valores da transformada de Fourier - foi definida em 1.000, e os valores com diferença superior a esse fator foram rejeitados (Zhou et al., 2015).

A transformação HLS foi utilizada para ilustrar o ciclo fenológico da cana-de-açúcar, no período de outubro a março em cada safra, tendo-se baseado nos termos harmônicos zero e primeiro, que representam a média e a amplitude do ciclo de seis meses dos índices de vegetação. Para cada pixel das séries temporais decompostas de EVI e NDVI, a fase do ciclo de seis 
meses foi atribuída à componente matiz, a amplitude do ciclo de seis meses à componente luminosidade, e a média do ciclo de seis meses à componente saturação. $\mathrm{Na}$ representação HLS resultante, aplicou-se o realce de contraste linear, para destacar pequenas diferenças e facilitar a interpretação visual. Ao final, realizou-se o mascaramento com os dados vetoriais do Canasat, referente ao mapeamento da distribuição espacial da cultura da cana-de-açúcar na região Centro-Sul do Brasil, por meio de técnicas de segmentação e interpretação visual de imagens tipo Landsat, de excelente qualidade temática (Rudorff et al., 2010). Este procedimento garantiu que a análise deste estudo fosse feita apenas sobre áreas de cultivo da cana-de-açúcar no Estado de São Paulo.

As representações HLS foram elaboradas com os termos harmônicos de EVI e NDVI para o Estado de São Paulo, respectivamente, no período de outubro a março das oito safras (Figuras 3 e 4). Para auxiliar a compreensão das representações HLS, a legenda de cores dessas figuras estabelece a correspondência com a ocorrência do pico vegetativo nas diferentes safras, o que dá uma impressão rápida das mudanças das regiões de cultivo da cana-de-açúcar. Neste tipo de representação HLS, uma alta intensidade referese a um EVI ou NDVI constantemente elevado, durante o período de seis meses, como em regiões de produção agrícola. As áreas de coloração mais clara apresentam um forte efeito do ciclo de seis meses. Como o matiz indica o tempo de máximo EVI ou NDVI, a ordem das cores vermelho amarelo/ verde/ ciano/ azul/ magenta - corresponde aproximadamente à temporização da fase nos meses de outubro/ novembro/ dezembro/ janeiro/ fevereiro/ março, respectivamente.

A intensidade brilhante de cinza-claro à branca refere-se a um EVI ou NDVI elevado durante o ciclo vegetativo, que se manifesta em todos os harmônicos, como nas regiões de Ribeirão Preto e Bauru, onde prevalece a intensificação do cultivo da cana-de-açúcar. O cultivo também pode ser observado predominantemente em tons de azul, com pico vegetativo que ocorre em fevereiro nas áreas de forte expansão, localizadas nas regiões de São José do Rio Preto e Araçatuba.

Os dados de precipitação acumulada do Estado de São Paulo, de outubro a setembro das safras 2004/2005 a 2011/2012, foram espacializados para representar a distribuição generalizada das chuvas (Figura 5). $\mathrm{Na}$ análise comparativa, realizada a seguir para a caracterização da média histórica da precipitação acumulada, utilizou-se a última normal climatológica de 30 anos, referente ao período de 1961 a 1990.

As safras 2004/2005, 2006/2007, 2007/2008 e 2008/2009 foram caracterizadas como sendo de acumulados de chuvas próximos aos das médias históricas (Climanálise, 2005-2012), ou seja, de condições bastante favoráveis ao desenvolvimento da cana-de-açúcar (Casagrande \& Vasconcelos, 2010), em termos de quantidade acumulada e distribuição das chuvas ao longo do ciclo vegetativo (Figura 5). É por essa razão que, nas representações HLS do Estado de São Paulo desses respectivos anos (Figuras 3 e 4), são observadas áreas de cultivo da cana-de-açúcar onde predominam tons de azul e de cinza-claro a branco, com pico vegetativo que ocorre normalmente em fevereiro, durante a fase de crescimento, em que há o alongamento dos colmos e o acúmulo de matéria seca da planta, que resultam na produção da cana-de-açúcar.

As safras 2010/2011 e 2011/2012 foram caracterizadas pelo acúmulo de precipitação abaixo da média histórica e pela má distribuição das chuvas (Climanálise, 2005-2012) ao longo do ciclo vegetativo (Figura 5). Isto acarretou baixas condições de umidade e elevação das temperaturas - desfavoráveis ao crescimento da cana-de-açúcar -, porque o alongamento dos colmos pode ser reduzido ou paralisado, o que pode adiantar a maturação que corresponde à senescência da planta, quando os produtos da fotossíntese são destinados principalmente ao armazenamento de sacarose nos colmos e, por consequência, obrigar à antecipação da colheita (Casagrande \& Vasconcelos, 2010).

As safras 2005/2006 e 2009/2010 foram marcadas pelos acumulados de chuva bem acima da média histórica (Climanálise, 2005-2012), distribuídos ao longo do ciclo vegetativo (Figura 5). O excesso de umidade, causado pelo grande volume de chuvas, prejudica muito a qualidade da cana-de-açúcar quanto ao armazenamento de sacarose nos colmos e pode acarretar o atraso da colheita, fazendo com que a cana madura permaneça no campo para ser colhida na próxima safra, o que é conhecido por "cana bisada", a qual passará por um novo ciclo de desenvolvimento,

Pesq. agropec. bras., Brasília, v.51, n.11, p.1868-1879, nov. 2016 DOI: $10.1590 / \mathrm{S} 0100-204 X 2016001100009$ 

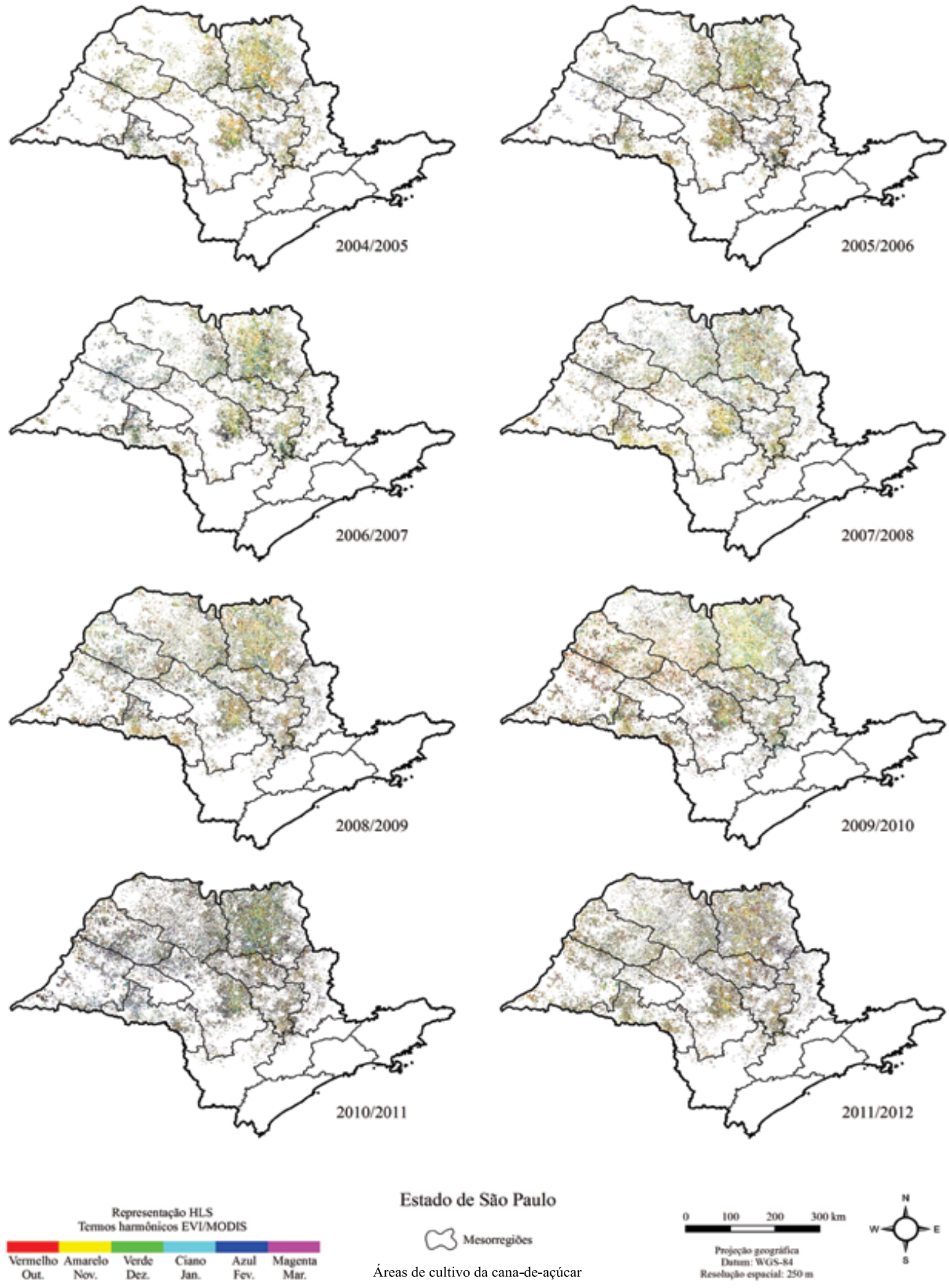

Figura 3. Representação HLS (hue, lightness, saturation) dos termos harmônicos de EVI de oito safras de cana-de-açúcar. 

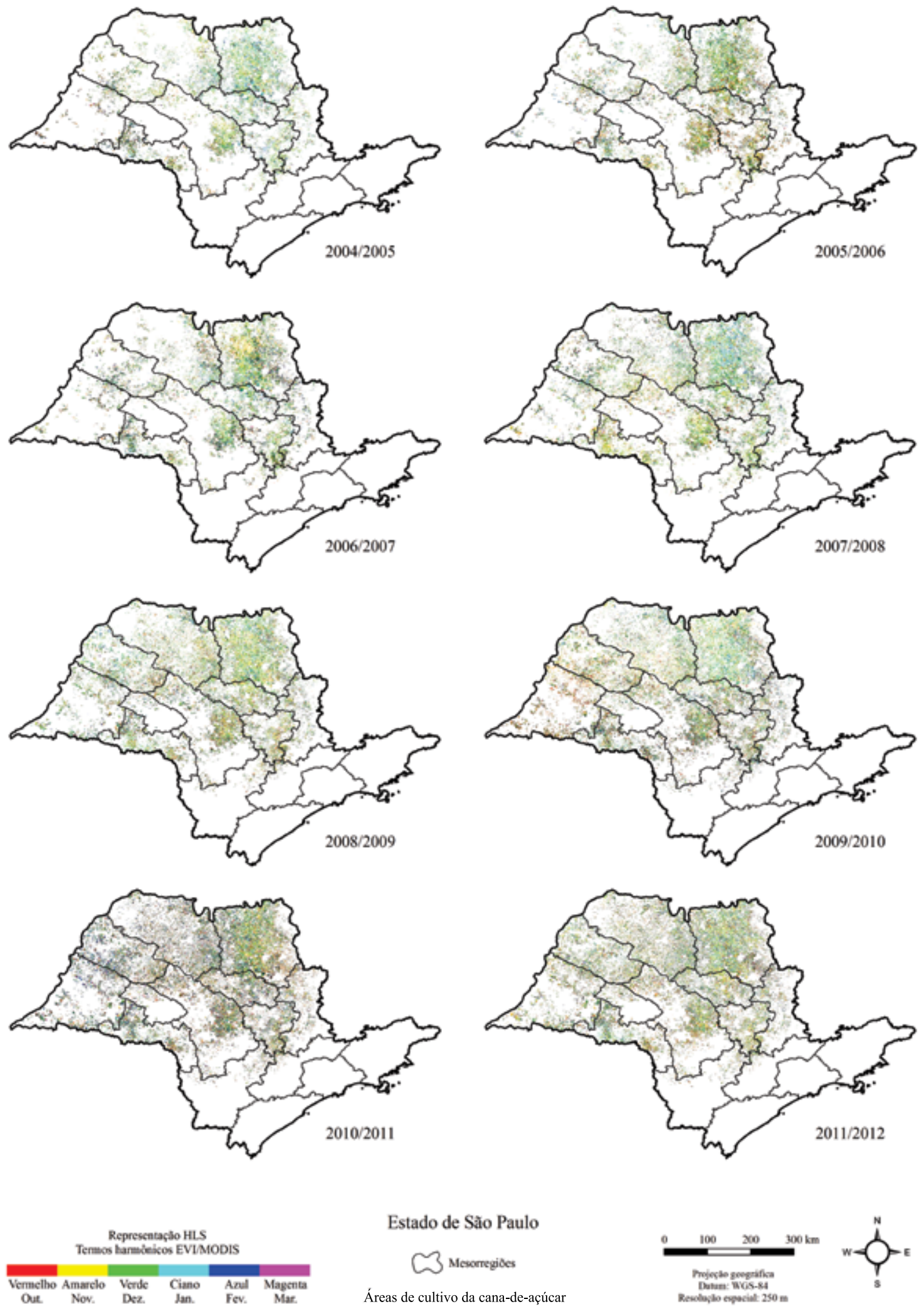

Figura 4. Representação HLS (hue, lightness, saturation) dos termos harmônicos de NDVI de oito safras de cana-de-açúcar. 

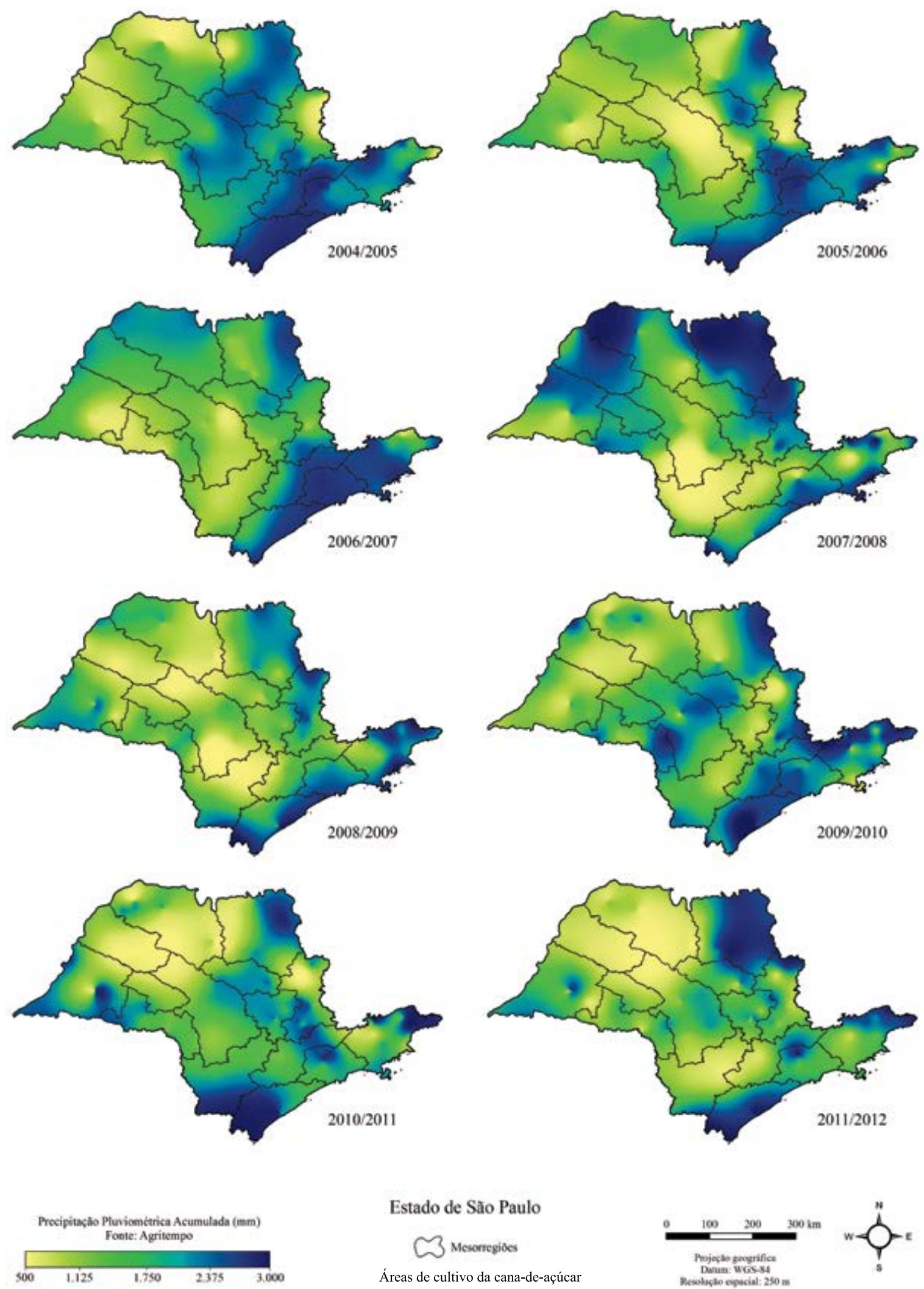

Figura 5. Mapas de precipitação pluvial acumulada de oito safras de cana-de-açúcar. 
porém com menor potencial de produção por ter mais brotos laterais (Casagrande \& Vasconcelos, 2010). Em razão das condições climáticas dessas safras, nas representações HLS desses referidos anos (Figuras 3 e 4), prevalecem os tons ciano, o que indica que o pico vegetativo foi adiantado para janeiro.

Como dito anteriormente, uma das principais vantagens que as imagens do sensor Modis apresentam é a alta repetitividade de extensas áreas da superfície terrestre, aliada à boa qualidade radiométrica e excelente precisão geométrica. Essas são características fundamentais para o estudo de alvos com grande dinâmica espectral e temporal e, assim, podem ser empregadas no monitoramento ao longo das fases do ciclo de desenvolvimento da canade-açúcar, desde o plantio ou rebrota até a colheita, em níveis regional e local (Aguiar et al., 2008; Eberhardt et al., 2015).

A abordagem espectrotemporal tem sido apontada como a mais apropriada para monitorar e mapear culturas agrícolas por meio da análise do comportamento dos pixels das imagens, ao longo de uma série temporal, com ênfase nas variações espectrais. Assim, é possível identificar a dinâmica da vegetação em distintas escalas temporais, acompanhando-se as variações dos ciclos fenológicos e caracterizando-se a transição entre diferentes usos e coberturas do solo ao longo dos anos (Ramme et al., 2010; Arenas-Toledo \& Epiphanio, 2011).

A metodologia proposta no presente trabalho mostrou que o potencial da análise harmônica para o estudo do comportamento cíclico dos índices de vegetação serve como base para uma análise exploratória espacial preliminar da série temporal Modis. O entendimento da dinâmica temporal é importante para evidenciar alterações do cultivo da cana-de-açúcar que é influenciado pela precipitação pluvial no Estado de São Paulo.

A representação HLS dos termos harmônicos é uma ferramenta útil e eficaz para a interpretação visual dos ciclos da vegetação, pois proporciona um efeito de transição suave entre os ciclos. Em estudos futuros, sugere-se utilizar também o segundo termo harmônico na representação HLS, com o propósito de se obter uma análise mais detalhada da variabilidade espacial sazonal relacionada às condições de manejo da cana-de-açúcar.

\section{Conclusões}

1. As representações HLS dos termos harmônicos das séries temporais de índices de vegetação Modis indicam que o cultivo da cana-de-açúcar no Estado de São Paulo apresenta padrões espaciais coerentes com o ciclo vegetativo e consistentes com a variabilidade das precipitações pluviais sazonais que afetam diretamente o momento de máximo do EVI e NDVI.

2. O pico vegetativo da cana-de-açúcar ocorre durante a fase de crescimento, em anos de chuvas normais, porém, em anos de pouca chuva, a fase de maturação da cultura é antecipada e, em anos de muita chuva, a fase de crescimento é antecipada, o que causa o atraso da maturação.

\section{Referências}

ADAMI, M. Estimativa da data de plantio da soja por meio de séries temporais de imagens Modis. 135p. Tese (Doutorado) Instituto Nacional de Pesquisas Espaciais, São José dos Campos, 2010 .

ADAMI, M.; MELLO, M.P.; AGUIAR, D.A.; RUDORFF, B.F.T.; SOUZA, A.F. de. A Web platform development to perform thematic accuracy assessment of sugarcane mapping in South Central Brazil. Remote Sensing, v.4, p.3201-3214, 2012. DOI: 10.3390/rs4103201.

AGRITEMPO: sistema de monitoramento agrometeorológico. Disponível em: <https://www.agritempo.gov.br/agritempo/ index.jsp>. Acesso em: 30 maio 2016.

AGUIAR, D.A.; RUDORFF, B.F.T.; RIZZI, R.; SHIMABUKURO, Y.E. Monitoramento da colheita da canade-açúcar por meio de imagens Modis. Revista Brasileira de Cartografia, v.60, p.375-383, 2008.

ANJOS, I.A. dos; FIGUEIREDO, P.A.M. de. Aspectos fitotécnicos do plantio. In: DINARDOMIRANDA, L.L.; VASCONCELOS, A.C.M. de; LANDELL, M.G. de A. (Ed.). Cana-de-açúcar. Campinas: IAC, 2010. 882p.

ANTUNES, J.F.G.; ESQUERDO, J.C.D.M.; LAMPARELLI, R.A.C.; RODRIGUES, L.H.A. Analysis of the vegetation phenology from the Alto Paraguai Basin throught the representation of harmonic cycles of EVI/Modis time-series. Geografia, v.38, p.109-122, 2013. Número especial.

ARENAS-TOLEDO, J.M.; EPIPHANIO, J.C.N. Harmonic amplitudeterms mask to highlight agriculture in the savanna domain below the Brazilian Amazonian frontier. International Journal of Remote Sensing, v.32, p.5021-5034, 2011. DOI: 10.1080/01431161.2010.495096.

BROWN, J.C.; JEPSON, W.E.; KASTENS, J.H.; WARDLOW, B.D.; LOMAS, J.M.; PRICE, K.P. Multitemporal, moderatespatial-resolution remote sensing of modern agricultural production and land modification in the Brazilian Amazon. 
GIScience and Remote Sensing, v.44, p.117-148, 2007. DOI: 10.2747/15481603.44.2.117.

BROWN, J.C.; KASTENS, J.H.; COUTINHO, A.C.; VICTORIA, D. de C.; BISHOP, C.R. Classifying multiyear agricultural land use data from Mato Grosso using time-series Modis vegetation index data. Remote Sensing of Environment, v.130, p.3950, 2013. DOI: 10.1016/j.rse.2012.11.009.

CASAGRANDE, A.A.; VASCONCELOS, A.C.M. de. Fisiologia da parte aérea. In: DINARDOMIRANDA, L.L.; VASCONCELOS, A.C.M. de; LANDELL, M.G. de A. (Ed.). Cana-de-açúcar. Campinas: IAC, 2010. 882p.

CLIMANÁLISE: boletim de monitoramento e análise climática, v.2027, 20052012. Disponível em: <http://climanalise.cptec. inpe.br/ rclimanl/boletim/>. Acesso em: 30 maio 2016.

EBERHARDT, I.D.R.; LUIZ, A.J.B.; FORMAGGIO, A.R.; SANCHES, I.D. Detecção de áreas agrícolas em tempo quase real com imagens Modis. Pesquisa Agropecuária Brasileira, v.50, p.605-614, 2015. DOI: 10.1590/S0100204X2015000700010.

EMBRAPA INFORMÁTICA AGROPECUÁRIA. Banco de Produtos Modis na Base Estadual Brasileira. Disponível em: $<$ https:/www.Modis.cnptia.embrapa.br/geonetwork/ srv/pt/ main.home>. Acesso em: 30 maio 2016.

ESQUERDO, J.C. D.M.; ANTUNES, J.F.G.; ANDRADE, J.C. de. Desenvolvimento do Banco de Produtos Modis na Base Estadual Brasileira. Campinas: Embrapa Informática Agropecuária, 2010. (Embrapa Informática Agropecuária. Comunicado técnico, 100).

FOLEY, J.D.; VAN DAM, A. Fundamentals of interactive computer graphics. Reading: Addison-Wesley, 1984. 664p.

FREITAS, R.M. de; ARAI, E.; ADAMI, M.; FERREIRA, A.S.; SATO, F.Y.; SHIMABUKURO, Y.E.; ROSA, R.R.; ANDERSON, L.O; RUDORFF, B.F.T. Virtual laboratory of remote sensing time series: visualization of Modis EVI2 data set over South America. Journal of Computational Interdisciplinary Sciences, v.2, p.57-68, 2011. DOI: 10.6062/ jcis.2011.02.01.0032.

HALLBEYER, M. IHS corepresentation of circular and noncircular variables using harmonic analysis parameters. Canadian Journal of Remote Sensing, v.33, p.416-421, 2007. DOI: $10.5589 / \mathrm{m} 07038$.

HUETE, A.; JUSTICE, C.; LIU, H. Development of vegetation and soil indices for ModisEOS. Remote Sensing of Environment, v.49, p.224-234, 1994. DOI: 10.1016/00344257(94)900183.

JAKUBAUSKAS, M.E.; LEGATES, D.R.; KASTENS, J.H. Crop identification using harmonic analysis of time-series AVHRR NDVI data. Computers and Electronics in Agriculture, v.37, p.127-139, 2002. DOI: 10.1016/S01681699(02)001163.

JAKUBAUSKAS, M.E.; LEGATES, D.R.; KASTENS, J.H. Harmonic analysis of time-series AVHRR NDVI data. Photogrammetric Engineering and Remote Sensing, v.67, p.461-470, 2001.

JIA, L.; SHANG, H.; HU, G.; MENENTI, M. Phenological response of vegetation to upstream river flow in the Heihe Rive basin by time series analysis of Modis data. Hydrology and
Earth System Sciences, v.15, p.1047-1064, 2011. DOI: 10.5194/ hess1510472011.

LATORRE, M.L.; SHIMABUKURO, Y.E.; ANDERSON, L.O. Sensor Modis: produtos para ecossistemas terrestres MODLAND. In: RUDORFF, B.F.T.; SHIMABUKURO, Y.E.; CEBALlOS, J.C. (Org.). O Sensor Modis e suas aplicações ambientais no Brasil. São José dos Campos: Parêntese, 2007.

RAMME, F.L.P.; LAMPARELLI, R.A.C.; ROCHA, J.V. Perfis temporais NDVI Modis, na canasoca, de maturação tardia. Engenharia Agrícola, v.30, p.480-494, 2010. DOI: 10.1590/ S010069162010000300012.

ROERINK, G.J.; MENENTI, M.; VERHOEF, W. Reconstructing cloudfree NDVI composites using Fourier analysis of time series. International Journal of Remote Sensing, v.21, p.19111917, 2000. DOI: 10.1080/014311600209814.

ROUSE, J.W.; HAAS, R.H.; SCHELL, J.A.; DEERING, D.W. Monitoring vegetation systems in the great plains with ERTS. In: EARTH RESOURCES TECHNOLOGY SATELLITE1 SYMPOSIUM, 3., 1973, Washington. Proceedings. Washington: NASA, 1974. v.1, p.309-317.

RUDORFF, B.F.T.; AGUIAR, D.A.; SILVA, W.F.; SUGAWARA, L.M.; ADAMI, M.; MOREIRA, M.A. Studies on the rapid expansion of sugarcane for ethanol production in São Paulo State (Brazil) using Landsat data. Remote Sensing, v.2, p.1057-1076, 2010. DOI: 10.3390/rs2041057.

SCARPARI, M.S.; BEAUCLAIR, E.G.F. de. Anatomia e botânica. In: DINARDOMIRANDA, L.L.; VASCONCELOS, A.C.M. de; LANDELL, M.G. de A. (Ed.). Cana-de-açúcar. Campinas: IAC, 2010. 882p.

SOARES, J.V.; BATISTA, G.T; SHIMABUKURO, Y.E. Sensor Modis: Histórico e Descrição. In: RUDORFF, B.F.T.; SHIMABUKURO, Y.E.; CEBALlOS, J.C. (Org.). O Sensor Modis e suas aplicações ambientais no Brasil. São José dos Campos: Parêntese, 2007.

UNICA. União da Indústria de Cana-de-Açúcar. UNICADATA. Disponível em: <http://www.unicadata.com.br/>. Acesso em: 30 maio 2016.

VERBESSELT, J.; HYNDMAN, R.; NEWNHAM, G.; CULVENOR, D. Detecting trend and seasonal changes in satellite image time series. Remote Sensing of Environment, v.114, p.106-115, 2010. DOI: 10.1016/j.rse.2009.08.014.

VICENTE, L.E.; GOMES, D.; VICTORIA, D. de C.; GARÇON, E.A.M.; BOLFE, E.L.; ANDRADE, R.G.; SILVA, G.B.S. da. Séries temporais de NDVI do sensor SPOT Vegetation e algoritmo SAM aplicados ao mapeamento de cana-de-açúcar. Pesquisa Agropecuária Brasileira, v.47, p.1337-1345, 2012. DOI: $10.1590 / \mathrm{S} 0100204 X 2012000900019$.

WARDLOW, B.D.; EGBERT, S.L.; KASTENS, J.H. Analysis of time-series Modis $250 \mathrm{~m}$ vegetation index data for crop classification in the U.S. Central Great Plains. Remote Sensing of Environment, v.108, p.290-310, 2007. DOI: 10.1016/j. rse.2006.11.021. 
WIT,A.de;SU,B.Derivingphenologicalindicators fromSPOTVGT data using the HANTS algorithm. In: INTERNATIONAL SPOTVEGETATION USERS CONFERENCE, 2., 2004, Antwerp. Proceedings. Belgium: [s.n.], 2005. p.195-201.
ZHOU, J.; JIA, L.; MENENTI, M. Reconstruction of global Modis NDVI time series: performance of harmonic analysis of time series (HANTS). Remote Sensing of Environment, v.163, p.217-228, 2015. DOI: 10.1016/j.rse.2015.03.018.

$\overline{\text { Recebido em } 20 \text { de outubro de } 2014 \text { e aprovado em } 13 \text { de junho de } 2016}$ 